\title{
RÉMI JARDAT
}

ISTEC École supérieure de commerce et de marketing Cnam-Lirsa

\section{« Pour Piketty » Une nouvelle pensée des Lumières pour les sciences humaines et de gestion}

Cet article ${ }^{1}$ vise à aider les managers comme les chercheurs en gestion à prendre la juste mesure scientifique et sociétale du désormais célèbre travail de Piketty ${ }^{2}$. Il confronte les critiques francophones actuelles à une lecture approfondie du « sytème » Piketty, et propose les premières leçons pour les sciences de gestion. Enfin, le positionnement de Thomas Piketty par rapport au marxisme est spécifié et argumenté sur des bases scientifiques et épistémologiques.

1. Une première version de cet article «point de vue » a été rédigée pour un public anglophone en mai 2014 et publiée dans Society and Business Review en octobre 2014. Nous soumettons ici une version amendée et complétée pour le public francophone, tenant notamment compte de la réception, tardive mais réelle, de la première édition française de septembre 2013.

2. Le capital au $X X I^{e}$ siècle, Thomas Piketty (2013). 


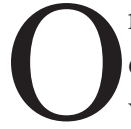
$\mathrm{n}$ peut publier une avancée majeure de ses travaux sous forme d'ouvrage et en retirer un impact considérable: Piketty l'a fait avec l'écho que l'on sait, ce qui constitue une leçon, certes mineure, mais néanmoins pertinente pour la communauté des chercheurs en gestion soucieux de leur utilité sociétale.

Comme beaucoup d'œuvres françaises majeures ces trente dernières années (que l'on songe à celles Latour ou Michel Serres, par exemple) Le capital au XXI siècle a rencontré localement un écho de magnitude et de qualité relativement faibles, jusqu'à ce que le public américain s'en empare et provoque, par un effet de ressac dont notre discipline est encore plus coutumière, un intérêt mérité dans le champ francophone. L'une des premières recensions sérieuses rédigées en français est due à un collègue de gestion (Zimnovitch, 2014), ce qui est plutôt à l'honneur de la discipline. Notre collègue de la revue Comptabilité Contrôle Audit restitue les grandes lignes de l'ouvrage et en retire notamment quelque éclairage pour les enjeux de transparence comptable propre à sa discipline. L'économiste Robert Boyer (2013/2014) a quant à lui publié un article approfondi sur l'ouvrage, avant que la Revue de la Régulation ne mette en ligne une version française disponible en accès libre. Marquées par l'emplacement disciplinaire de leur auteur, ces critiques principalement élogieuses mettent en avant les champs de recherche complémentaires qu'ouvre ce travail aux économistes : analyser en contrepoint les conflits de distribution autour du capital salarial, pousser plus avant la théorisation proprement économique que suggèrent les résultats empiriques de Piketty, développer un synoptique consistant des différentes dépendances et rétroactions entre les variables mesurées sur la longue durée pour consolider la valeur prospective des conclusions avancées par l'auteur, non sans une comparaison entre le destin possible de ces travaux et celui que connut la théorie de la régulation.

L'intérêt de notre propos consiste à entreprendre, pour les sciences de gestion en général et dans une optique entreprises et sociétés, ce que Robert Boyer a effectué pour la discipline économique : exposer et critiquer le cœur scientifique et épistémologique du travail de Piketty pour tenter d'éclairer en retour les enjeux de la recherche en gestion ${ }^{3}$.

La lecture de cet ouvrage long (1000 pages) mais jamais ennuyeux est un choc de dépaysement : le monde qui est le nôtre et qui sera probablement celui de nos deux prochaines générations de descendants y apparaît sous un jour entièrement nouveau et quelque peu sinistre. La raisons de ce choc n'est hélas qu'une trop bonne raison : c'est la conjonction de propositions et de prévisions radicales et d'une démonstration désespérément rigoureuse fondée sur une base de données immense qui tire sa robustesse d'un vaste effort collectif et international. En mode mineur, l'auteur propose également quelques développement de

\footnotetext{
3. J'ai pour ma part commencé ce travail en septembre 2013 et pris date dès ce moment pour faire un exposé critique de l'ouvrage lors d'un colloque qui s'est tenu à Milan le 8 mai 2014. La réaction passionnée des collègues de gestion provenant de 50 nationalités différentes, ainsi que le succès éruptif de l'ouvrage Outre-Atlantique, m'ont incité à publier ce travail dans sa langue de rédaction originelle, d'en proposer une adaptation française au rédacteur en chef de cette revue lors des états-généraux du management le 22 mai 2014. Qu'il soit remercié sans réserve pour la confiance ainsi accordée.
} 
grand intérêt pour tous les chercheurs en sciences humaines et notamment le champ des sciences de gestion

On peut faire le pronostic que l'onde de choc créée par cet ouvrage sera pérenne et, si certaines des préoccupations de l'auteur aussi bien que sa critique de Ricardo et Marx, comme le titre de l'ouvrage luimême, font écho à l'opus magnum de Marx, il faut être clair sur un point (que nous allons démontrer plus loin) : le travail de Piketty est tout sauf marxiste, n'en déplaise à certain inspecteur des finances au verbe haut mais superficiel (Baverez, 2013).

Plus qu'un penseur du soupçon, Piketty doit être au contraire rapproché des philosophes des Lumières, en ce qu'il se veut fondateur d'une nouvelle raison démocratique qui croit aux vertus du débat explicite au lieu de n'y voir qu'une supercherie bourgeoise à l'instar de la tradition marxiste. En cela il s'inscrit dans le renouvellement d'une pensée post-libérale initié par Pierre Rosanvallon avec le lancement de la collection La République des Idées.

Dans un premier temps, nous rappelons les principales propositions de Piketty et montrons à quel point, en dépit de leur lourd ancrage empirique, elles font « système », même s'il ne s'agit pas d'un système aussi fermé que le voudrait une certaine tradition économiste. Dans un deuxième temps, nous soulignons la dimension « cosmologique » de l'ouvrage par laquelle Piketty, non seulement soulève des défis touchant à la création et à la répartition des revenus, mais encore remet en cause notre conception de ce qu'est et devrait être la vie bonne dans des sociétés démocratiques et méritocratiques. Enfin, et cela ne manquera pas de faire écho pour le champ des sciences de gestion, nous analysons les positions épistémologiques prises par Piketty, ce qui permet d'en dégager une juste perspective par rapport au champ marxiste et cryptomarxiste. Nous en concluons que Piketty est plus un homme des Lumières qu'un penseur du soupçon, en ce qu'il pose la question «sagittale » (pour reprendre le commentaire de Kant par Foucault, 1984) d'une humanité devenue suffisamment adulte pour s'autogouverner par la raison : «Qui sommes-nous?»

\section{I - LA THÈSE PRINCIPALE DE PIKETTY : LA MACHINE INFERNALE À CONCENTRER LE CAPITAL ET CAPTURER LES REVENUS}

Loin de ne théoriser que les inégalités, Piketty a également renouvelé la théorie de la richesse, sur la base de résultats empiriques solides; cette théorie écrit les mécanismes par lesquels croissance et concentration du capital sont liés par des rétroactions positives que ne vient modérer aucun mécanisme de marché. Grâce à des statistiques très soigneuses de revenu et de richesse sur le long terme, il prouve empiriquement que l'euthanasie de la rente et de l'héritage au bénéfice du mérite par le travail n'était qu'illusion, produite par la conjonction de deux facteurs de natures très différentes.

\section{L'illusion méritocratique et les invisibles statistiques}

Le premier facteur d'illusion est la conjoncture historique et démographique très particulière du $\mathrm{XX}^{\mathrm{e}}$ siècle, qui a créé des chocs majeurs détruisant le stock de capital et ainsi « rajeunissant» la structure économique. La preuve hélas incontestable est donnée que les résultats plutôt rassurants et 
socialement apaisants de Solow et Kuznets ne sont rien de plus que l'effet d'un biais d'échantillonnage temporel sur la répartition et l'évolution du stock de capital. Ces conditions très spécifiques n'étant pas susceptibles de se reproduire au cours de notre siècle, il est très vraisemblable que la reproduction et la croissance y seront très stables. La production par tête devrait s'égaliser de plus en plus de par le monde, parce que la frontière technologique sera atteinte par toutes les sociétés en même temps que la démographie se stabilisera. Il n'y aura ainsi plus aucun de ces phénomènes de rattrapage sans lesquels la croissance ne peut dépasser en moyenne les 1-2 \% par an. Certes, Piketty explique qu'une croissance faible mais non nulle produit des effets très différents de la stagnation économique totale des sociétés d'antan, et permet notamment un certain degré de transformation sociale. Cela n'est néanmoins possible que si des dispositifs institutionnels bien conçus (et notamment étatiques) sont mis en place, faute de quoi un mécanisme naturel de concentration $\mathrm{du}$ capital se produira sans limite aucune dans un monde aussi stable, au point qu'il devient raisonnable de se demander si, à la fin du siècle, le monde ne sera pas possédé par une poignée de personnes fortunées (pour certaines via des institutions paravent comme un État pseudo-communiste). Ainsi, à la fin du $\mathrm{XXI}^{\mathrm{e}}$ siècle, 99,99\% de nos enfants pourraient travailler pour l'émir du Qatar ou pour quelques oligarques chinois. Le second facteur d'illusion est d'ordre méthodologique. Les indices et outils statistiques couramment utilisés pour mesurer les inégalités sont totalement inadéquats pour percevoir la tendance lourde à la concentration des revenus, et surtout, de la richesse accumulée. La richesse (ou capital) accumulée se concentre à un tel degré que les indicateurs usuels ne détectent pas cette concentration, en particulier parce qu'ils découpent l'échelle des revenus et des richesses uniquement à l'échelle du décile, alors que seule la mesure des premiers centiles et, de plus en plus, des premiers millimes de la population offre la mesure de ce phénomène.

\section{La machine infernale du capital et de la richesse}

Grâce à des statistiques d'un périmètre et d'une fiabilité inédites (quoi qu'en disent des journalistes britanniques non qualifiés en matière de calibrage statistique, voir Chavagneux et Jeanneau, 2014, p. 67), fondés notamment sur les statistiques fiscales et les successions, Piketty montre que son modèle très simple de concentration du capital et de partage entre revenus du travail et revenus du capital n'est pas qu'une élégante construction théorique, mais aussi un descripteur et prédicteur pertinent et fiable des réalités économiques et sociales. Sans revenir sur l'appareil empirique fourni dans l'ouvrage et sur le site internet associé, nous nous contentons d'exposer ici les rouages clés de ce qu'il faut bien appeler une « machine infernale » décrite dans parties I et II de l'ouvrage.

\section{Deux changements de variables affectant les paramètres-clés des débats économiques actuels : le revenu et le capital}

«Par définition, le revenu national mesure l'ensemble des revenus dont disposent les résidents d'un pays donné, quelle que soit la forme juridique que prennent ces revenus » (p. 79). Il faut bien noter ici que Piketty calcule le revenu national en soustrayant 
de l'habituel PIB la dépréciation du capital et en y ajoutant les revenus nets provenant de l'étranger. Il fait ainsi glisser l'attention vers des variables à même de rendre compte des disparités qui persistent entre les personnes (celles qui vivent de leur travail par rapport à celles qui vivent de leurs rentes) aussi bien qu'entre les nations (car il y a de même des nations globalement laborieuses et des nations beaucoup plus rentières vis-à-vis des autres). Ce faisant, Piketty se donne les moyens de mesurer des déterminants réalistes des rapports de force entre les personnes et entre les peuples, souvent ressenties mais jamais clairement explicitées ni mesurées jusqu'ici.

De même, Piketty propose une définition tout aussi pertinente et lourde de conséquences du concept de capital: «Dans le cadre de ce livre, le capital est défini comme l'ensemble des actifs non humains qui peuvent être possédés et échangés sur un marché. » (p. 82). En conséquence logique, le terme « capital » englobe toutes les formes de biens appropriables (et notamment immobiliers) aux côtés des capitaux financiers et industriels habituellement pris en compte dans les statistiques économiques, tandis que le concept de "capital humain » est révoqué comme nul et non avenu du fait de l'abolition de l'esclavage. La prise en compte du capital immobilier, trop souvent négligée par ailleurs, est ici mise au centre des calculs, et l'on se rend compte avec Piketty que l'immobilier a pris un poids énorme dans nos économies, trop souvent négligé : « le stock de capital dans les pays développés se partage actuellement en deux moitiés approximativement égales: capital logement d'une part, et capital productif utilisé par les entreprises et administrations d'autre part. » (p. 91).
Nous allons voir que ce second changement de variable est également d'importance majeure. Il semble que les critiques francophones (Boyer, 2013; Husson, 2014) n'aient pas voulu percevoir la portée de ce changement de variables. Loin d'oublier les conflits de répartition, Piketty les laisse à voir au contraire d'une manière inédite.

\section{Deux ratios clés : la part $\alpha$ des revenus du capital dans le revenu national (répartition entre revenus du travail et du capital) et le ratio $\beta$ du stock de capital sur le flux de revenu}

Le fait marquant à propos de $\alpha$ est sa forte croissance depuis les trente dernières années, aussi bien en Europe qu'aux ÉtatsUnis. Cela signifie, que, dans nos sociétés, une part de plus en plus grande de la richesse produite est captée par les détenteurs de capital. Aussi, l'un des enjeux clés du $\mathrm{XXI}^{\mathrm{e}}$ siècle est-il la possible montée de $\alpha$ jusqu'aux niveaux qu'il avait atteints à la Belle Époque. Revenir à une telle structure de revenus constituerait une rupture sociétale majeure pour la manière dont chacun donne aujourd'hui sens à sa position sociale, à son mérite, comme aux destins possibles offerts à ses enfants. Toute l'infrastructure psychologique de nos sociétés, méritocratique au moins dans ses principes, est en jeu : «La question de savoir s'il est justifié et utile pour la société que les détenteurs du capital reçoivent cette productivité marginale [du capital] comme rémunération de leur titre de propriété (et de leur épargne passée, ou bien de celle de leurs ancêtres), sans qu'aucun travail nouveau soit apporté, est évidemment une question centrale, sur laquelle nous aurons amplement l'occasion de revenir. » (p. 340).

Toutes choses égales par ailleurs, il est très vraisemblable que $\alpha$ va s'élever au fur et à 
mesure que le stock de capital, comparé au flux du revenu national, s'accroîtra de pair. Ce dernier phénomène est mesuré par le ratio $\beta$ du capital sur le revenu national. Ici encore le fait incontournable est que $\beta$, qui se situait à des niveaux très bas suite aux chocs externes du $\mathrm{XX}^{\mathrm{e}}$ siècle, est désormais très élevé : il atteint typiquement cinq à six années de revenu national dans les pays développés. D'un côté, avoir accumulé un tel stock de richesse peut être considéré comme l'une des réussites majeures de nos sociétés. En outre, explique malicieusement Piketty, cela donne les moyens de résoudre potentiellement les problèmes de la dette d'État, puisque celle-ci ne représente, en comparaison, qu'un an de revenu national. Malheureusement, la médaille a son revers : un $\beta$ très élevé signifie en même temps que seule une petite part de la richesse se renouvelle chaque année, ce qui tend à favoriser la domination des détenteurs de capital (souvent âgés) sur les personnes jeunes et actives. Cela ne peut pas être sans conséquence sur la dynamique des sociétés concernées. En outre, l'extrême concentration de ce stock de capital dans des mains privées accentue le problème. Par ailleurs, il est illusoire de penser que la technologie viendrait atténuer cette dynamique. Ainsi, explique Piketty : " La technologie moderne utilise toujours beaucoup de capital, et surtout la diversité des usages du capital fait que l'on peut accumuler énormément de capital sans que son rendement s'effondre totalement. »C'est donc une illusion de croire que « les actionnaires bedonnants auraient été remplacés par les cadres méritants, simplement par la grâce du changement technologique » (p. 354). Voilà un sujet de réflexion intéressant pour les chercheurs en ressources humaines, particulièrement en matière de motivation des cadres!

\section{Deux moteurs du changement : le rendement net du capital $r$ et la croissance de la production $g$}

Le capital a sa propre dynamique. Typiquement, le rendement net du capital, $r$, atteint une moyenne ${ }^{4}$ de 5 à $6 \%$ dans toute économie stable, alors que la production et le revenu croissent beaucoup plus lentement, au maximum à 1,5\%-2\% l'an en moyenne dans les pays où la frontière technologique a été atteinte. En conséquence, il devient impossible à la plupart des citoyens, aussi méritants soient-ils, de rattraper le niveau de revenu atteint par ceux qui ont bénéficié d'un gros héritage, ce qui fige la société : « Il suffit donc aux héritiers d'épargner une part limitée des revenus de leur capital pour que ce dernier s'accroisse plus vite que l'économie dans son ensemble. Dans ces conditions, il est presque inévitable que les patrimoines hérités dominent largement les patrimoines constitués au cours d'une vie de travail, et que la concentration du capital atteigne des niveaux extrêmement élevés, et potentiellement incompatibles avec les valeurs méritocratiques et les principes de justice sociale qui sont au fondement de nos sociétés démocratiques modernes. » (p. 53). Cette rétroaction positive constitue le cœur de la machine infernale du capital dans un monde stable, telle qu'elle a déjà régné auparavant au cours du $\mathrm{XIX}^{\mathrm{e}}$ siècle en France et au Royaume-Uni avant la Première Guerre mondiale. Il y a là une pro-

4. Les critiques un peu rapides ont manqué qu'il s'agissait d'une moyenne 
fonde force de divergence par laquelle une classe fortunée devient inexorablement de plus en plus déconnectée du reste de la société. À l'opposé, ceux qui naissent sans capital hérité, qu'il soit de nature industrielle ou immobilière, n'ont aucune chance de rejoindre l'élite, puisque la croissance est trop faible pour offrir des opportunités d'enrichissement licite. Pis encore, une bonne partie de leur propre revenu sera ponctionnée, sous forme de loyers (ou fiscalement pour rembourser les dettes d'État) qui viendront alimenter l'enrichissement des héritiers fortunés. La divergence entre détenteurs et non-détenteurs de capitaux est d'autant plus forte que, comme le suggèrent les séries statistiques, le rendement net du capital r s'accroît lui-même avec le volume de fortune détenue : les forts patrimoines peuvent en effet s'investir de manière à la fois plus riche et plus diversifiée, si bien que, plus les marchés financiers sont efficients, plus ils contribuent à la divergence entre les super-riches et le reste de l'humanité. On est bien loin du rêve entrepreneurial des étudiants de nos université et business schools : « Le capital n'est jamais paisible : il est toujours risqué et entrepreneurial, tout du moins à ses débuts; et en même temps il tend toujours à se transformer en rente dès lors qu'il s'accumule sans limite - c'est sa vocation, son destin logique. » (p. 187).

Une simple équation comptable : $\alpha=r x \beta$, combinée à la règle de convergence empirique observée $\beta=s / g$

Ces équations traduisent les conséquences macroéconomiques de la rétroaction positive entre capital et rente précédemment décrite. La première équation $\alpha=\mathrm{r} \times \beta$ exprime cette évidence comptable que la part des revenus du capital dans le revenu national est le produit du rendement $\mathrm{du}$ capital, r, par $\beta$, le ratio capital/revenu. Nous savons déjà que $r$ va très probablement demeurer à un niveau élevé et régulier de 4,5-5 \% minimum. La seconde équation prévoit en outre que $\beta$ lui aussi demeurera très élevé, dans un monde où l'épargne $\mathrm{s}$ sera élevée et la croissance $\mathrm{g}$ sera basse. Cela signifie que la part $\alpha$ des revenus du capital va s'accroître encore plus à long terme, en d'autres termes que le capital captera une part de plus en plus grande du revenu national au détriment du travail. Pour qui nos enfants travailleront-ils ? Probablement par pour eux, mais, pour la plus grande part, à enrichir les détenteurs de capital qui auront obtenu leur fortune par héritage et non en vertu de leurs mérites. Piketty y voit d'une sorte d'involution historique : «La planète entière pourrait ressembler d'ici à 2100 à l'Europe de la Belle Époque - tout du moins du point de vue de l'intensité patrimoniale » (p. 310).

\section{Deux résultats empiriques de longue durée, combinés avec des prévisions plutôt vraisemblables : $r-g>2,5 \%$ et $1,3<\sigma<1,6$ où $\sigma$ est l'élasticité de la substitution du capital au travail dans la fonction de production}

Les variables des équations précédentes ne sont pas indépendantes. En particulier, Marx et les économistes du $\mathrm{XX}^{\mathrm{e}}$ siècle avaient estimé que le rendement du capital $\mathrm{r}$ devrait naturellement diminuer au fur et à mesure que le stock de capital s'accroîtrait, en vertu d'une sorte de loi de l'offre et de la demande de capital. Malheureusement, selon Piketty, les données empiriques vont dans un sens tout à fait contraire : bien que $r$ soit susceptible de décroître modérément à l'avenir, cet effet sera plus que compensé 
par le gigantesque stock de capital accumulé, et il en résultera au final un partage des revenus toujours plus défavorable au travail. La preuve la plus évidente en est, selon Piketty, que l'élasticité de substitution du capital au travail est supérieure à 1. En outre, l'inégalité $r>g$ qui caractérise aujourd'hui l'Europe et les États-Unis devrait s'étendre à la planète entière vers 2050, avec un écart d'au moins 2,5\%, ce qui alimentera la machine infernale de la divergence entre héritiers fortunés et classes laborieuses, quels que soient les talents développés au sein de ces dernières. En conclusion, Piketty montre qu'une machine infernale, qui tournait à plein régime au $\mathrm{XIX}^{\mathrm{e}}$ siècle et a été provisoirement enrayée par les chocs du $\mathrm{XX}^{\mathrm{e}}$ siècle, s'est remise en marche depuis trois décennies à notre insu : la part du travail dans les revenus nationaux va décroître sans cesse tandis que le rapport capital/revenu se stabilisera à des niveaux très élevés. Le stock de capital, essentiellement privé, pourrait atteindre de neuf et dix ans de revenu national vers 2050. Cela signifie que nous et nos enfants vivront dans un monde dans lequel le passé pèsera extrêmement lourd sur l'économie et sur les statuts sociaux des uns et des autres. Un monde où la hiérarchie des fortunes héritées ne sera plus jamais remise en cause par le travail et le mérite, un monde dominé par une poignée d'héritiers fortunés face auxquels les pouvoirs publics et la classe politique n'auront presque aucun pouvoir, un monde privé de tout espoir méritocratique, un monde sans rien de commun entre les ultra riches et les gens ordinaires, ce qui veut dire un monde sans citoyenneté, un bouleversement majeur et inexorable de nos civilisations et régimes démocratiques, dont la venue était restée jusqu'ici inaperçue. C'est pourquoi nous pouvons affirmer que le travail de Piketty comporte une dimension cosmologique et eschatologique. D'où l'intérêt de l'analyser en termes de cosmologie et de le comparer à d'autres cosmologies enracinées dans l'économie, tel le Marxisme.

\section{II - UNE OEUVRE-MONDE ENTRE FUKUYAMA ET ROSANVALLON}

Pour la génération des Européens (de l'Ouest) formés entre 1945 et 1975, il allait de soi qu'une croissance forte et une réduction des inégalités n'étaient pas des événements temporaires mais les tendances lourdes d'un monde nouveau. De nombreuses populations, et parmi eux les intellectuels, avaient pour horizon eschatologique une société où les inégalités les plus criantes comme le mépris et l'arrogance des « bourgeois » et des « snobs » appartiendraient au passé. C'est à cette génération qu'appartiennent encore la plupart des savants célèbres en sciences humaines. Il est nouveau et d'autant plus intéressant de voir des chercheurs de la génération suivante (dite "génération $X$ ») démontrer qu'au contraire : «c'est la période des Trente Glorieuses qui était exceptionnelle» (p. 161), et cela pour une raison difficilement contestable empiriquement : "Il n'existe aucun exemple dans l'histoire d'un pays se trouvant à la frontière technologique mondiale et dont la croissance de la production par habitant soit durablement supérieure à 1,5\%.» (p. 156). Au contraire du siècle précédent, le monde qui vient s'annonce comme étant d'une relative stabilité, extrêmement favorable à l'accumulation et à la concentration du capital, où la production par tête sera devenue globalement homo- 
gène : « le niveau de production par habitant aurait dès 2050 rattrapé un peu partout le niveau des pays les plus riches, aussi bien en Chine qu'en Europe de l'Est, en Amérique du Sud, en Afrique du Nord et au MoyenOrient. À partir de cette date, la répartition de la production mondiale s'approcherait donc de celle de la population. » (p. 166).

\section{Un « Fukuyama de gauche $» . .$.}

Par certains aspects, la vision du monde développée par Piketty se rapproche de la Fin de l'Histoire prévue par Francis Fukuyama $(1989,1992)$ juste après la chute du monde soviétique. Aucun bouleversement externe n'est censé détruire le stock de capital accumulé ni entraver la convergence géographique de la production à l'échelle du monde. À l'opposé du républicain centriste Fukuyama, mais justement à l'opposé, un Piketty de gauche ne prévoit pas un radieux avenir libéral, mais plutôt une ère analogue au $\mathrm{XIX}^{\mathrm{e}}$ siècle conduite par la «machine infernale du capital » décrite plus haut. Le terme employé par Piketty est celui de divergence causée par l'extrême concentration de la propriété. En effet, une distribution relativement uniforme de la production par habitant de par le monde ne signifie aucunement que le revenu y soit accumulé de manière uniforme. Au contraire, le monde qui s'annonce verra le revenu créé par des milliards d'êtres humains capté et transféré vers des régions et des détenteurs de capitaux lointains, ce qui devrait générer de grands déséquilibres et des frustrations aiguës au sein des populations serves.

\section{2. ... avant tout inspiré par la pensée de Pierre Rosanvallon}

Mais Piketty n'est pas qu'un Fukuyama de gauche. Il est aussi un théoricien de la communalité (Rosanvallon, 2011, p. 382), par quoi nous entendons un chercheur profondément préoccupé par la valeur d'égalité définie par les philosophes des Lumières, une valeur qui sous-tend le sentiment qu'ont les citoyens, au sein des démocraties modernes, de vivre plus ou moins dans un monde commun, quelles que soient les inégalités économiques (modérées) qui persistent entre eux. On ne saurait trop insister sur le fait que le travail de Piketty complète dans une large mesure celui de Rosanvallon sur la démocratie et l'égalité. Les visions du monde des deux chercheurs s'emboitent ainsi l'une dans l'autre, le travail de Piketty pouvant être lu comme le pendant économique et prospectif de celui de Rosanvallon.

C'est tout d'abord le cas pour la face dystopique de chacune des deux visions développées de part et d'autre, où le XIX ${ }^{\mathrm{e}}$ siècle fait office de repoussoir principal. L'argumentation de Rosanvallon consiste ainsi, dans La société des égaux (2011), à montrer que les philosophes du XVIII ${ }^{\mathrm{e}}$ siècle, aussi bien français qu'écossais et américains, avaient construit leur concept d'égalité sans avoir la moindre idée de la divergence majeure qu'allait provoquer la Révolution Industrielle au siècle suivant. Cette divergence, typiquement décrite par le roman de Disraeli Sybil, or the two nations, a engendré des idéologies anti-démocratiques autant qu'elle fut grosse des tragédies du $\mathrm{XX}^{\mathrm{e}}$ siècle. En miroir, il est remarquable que Piketty non seulement illustre son propos par les romans de Honoré de Balzac et Jane Austen (ainsi que celui de Disraeli comme le fait Rosanvallon), mais encore considère lui aussi le $\mathrm{XIX}^{\mathrm{e}}$ siècle comme une régression, comparé à la période de la Révolution et de l'Empire : «Vue de la 
Restauration et de la monarchie de juillet, la période révolutionnaire et napoléonienne laissera ainsi un bon souvenir aux classes populaires. » (p. 356-357).

Piketty et Rosanvallon expriment également une même critique de l'" extrémisme méritocratique ». Rosanvallon dénonce ainsi un «principe de l'égalité des chances restreinte au sommet de la société [de Guizot, qui] a été le masque de la reproduction massive des places dans l'ensemble de la société » (p. 126). Piketty recourt au même genre d'argument à propos des revenus vertigineux des super-cadres des multinationales et parle lui aussi d'" extrémisme méritocratique » qu'il définit comme « un besoin des sociétés modernes, et en particulier de la société américaine, de désigner elles-mêmes les gagnants et de leur offrir des rémunérations d'autant plus extravagantes qu'ils semblent avoir été choisis en fonction de leur mérite propre, et non suivant les logiques inégalitaires du passé » (p. 530). Si les deux auteurs fustigent à un même degré les «super-salaires » des « super-cadres », chacun met en avant toutefois des arguments spécifiques. Piketty concentre sa critique sur les systèmes d'incitation, ce qui ne sera pas sans échos directs avec les recherches en gestion sur la gouvernance et le contrôle. L'inflation des salaires des dirigeants lui apparaît en effet comme une preuve de « la faillite de la gouvernance d'entreprise » (p. 531) et d'une certaine idéologie libérale ayant digéré pour son propre compte la Fable des abeilles de Mandeville prétendument reprise par Adam Smith : «Sans aller jusqu'à parler de la « main qui se sert dans la caisse », force est de constater que cette image est sans doute plus adaptée que celle de la «main invisible » (p. 524). De son côté, Rosanvallon s'exprime en termes plus politico-moraux en phase avec sa focalisation sur la légitimité démocratique (2011, p. 331-332) : ce ne sont pas la vertu, le mérite ou le talent individuel qui ont déterminé la montée en flèche des rémunérations les plus élevées - et ce d'autant moins que les échecs ne les ont souvent guère affectées. C'est, à des titres divers, ce qui relève de la ruse, de la manipulation, du rapport de force, de la connivence, voire de la corruption, qui a joué le rôle essentiel. » L'emboîtement complémentaire des deux argumentations est ici particulièrement flagrante.

Les argumentations de Rosanvallon et Piketty sont également en correspondance étroite dans leurs volets utopiques. Rosanvallon explique en 2011 (p. 22) que, pour redevenir crédible et robuste en regard des défis du $\mathrm{XXI}^{\mathrm{e}}$ siècle, l'idéal d'égalité doit être renouvelé en prenant en compte les forces de divergence économique, principalement à travers trois concepts qu'il dénomme "singularité, réciprocité et communalité ». Le concept de communalité est central dans la vision de Piketty, par la notion de divergence qui la dessine en quelque sorte en négatif, puisque cette dernière détruit la communalité, et, partant, la citoyenneté et la démocratie. La réciprocité est un second concept plus rarement mais clairement mis en avant par Piketty, par exemple lorsqu'il dessine les contours d'une possible utopie sociale de l'égalité : « Il n'est pas interdit d'imaginer une société idéale où toutes les autres tâches seraient presque totalement automatisées, et où chacun pourrait presque entièrement se consacrer à l'éducation, la culture et la santé, pour lui-même et pour les autres, où chacun serait le professeur, l'écrivain, l'acteur, le docteur de quelqu'un d'autre » (p. 487). 
Pour conclure, les argumentations de Piketty et Rosanvallon se rejoignent dans la conviction commune que (Rosanvallon, 2011, p. 353) : «Les inégalités, et c'est là le point essentiel, n'affectent donc pas seulement les plus défavorisés, elles ont un effet délétère pour tous. »

\section{III - L'ÉPISTÉMOLOGIE DE PIKETTY : CONTRE L'OBSCURANTISME D'UN CERTAIN MAINSTREAM ET LES OBSCURITÉS MARXISTES}

Ses collègues économistes l'ont relevé eux aussi, la pertinence de travaux de Piketty sur la richesse tient à l'originalité et à la crédibilité de sa méthode, qui vient combler un vide transdisciplinaire : «L'exploitation historique et statistique de la source fiscale tombe dans une sorte de "no man's land" académique, trop historique pour les économistes, et trop économique pour les historiens. » (p. 39). Le point de résonance le plus fort avec la discipline des sciences de gestion se trouve peut-être avec les attaques portées par Piketty sur les études quantitatives décontextualisées de ses collègues. Ses arguments semblent en effet porter jusque dans le débat entre les tenants et les critiques du mainstream en sciences de gestion.

\section{Contre l'obscurantisme d'un certain mainstream en sciences humaines}

Tout d'abord, critiquer l'abus des modélisations quantitatives en économie semble d'autant plus significatif, vu de la gestion, que l'économie est précisément censée avoir atteint un degré de maturité et de scientificité plus avancé que notre discipline : «La discipline économique n'est toujours pas sortie de sa passion infan- tile pour les mathématiques et spéculations purement théoriques, et souvent très idéologiques, au détriment de la recherche historique et du rapprochement avec les autres sciences sociales. Trop souvent, les économistes sont avant tout préoccupés par de petits problèmes mathématiques qui n'intéressent qu'eux-mêmes, ce qui leur permet de se donner à peu de frais des apparences de scientificité et d'éviter d'avoir à répondre aux questions autrement plus compliquées posées par le monde qui les entoure. » (p. 63)

Piketty concentre notamment ses critiques sur les théories dites «à agent représentatif » (la théorie de l'agence fréquemment mobilisée en gestion entre dans cette catégorie), parce qu'elles occultent totalement la réalité des différences et diversités sociales, écartant ainsi du champ scientifique les problèmes les plus importants : «l'autre raison pour laquelle on a trop longtemps négligé la question de la répartition des richesses est un goût excessif de la profession pour les modèles mathématiques simplistes dits "à agent représentatif" "(p. 38). En particulier, les insuffisances théoriques de Ricardo et Keynes sont diagnostiquées comme les conséquences du recours à ce type de théorie. Du côté des sciences de gestion, on peut y voir aussi bien une mise en garde contre le recours général aux modèles de la psychologie quantitative, avec leurs tests de construits considérés comme étant l'alpha et l'oméga (ou plutôt l'alpha et le rhô) de toute recherche sérieuse. La psychologie quantitative repose sur l'hypothèse qu'une certaine typologie est une bonne manière de décrire l'espèce humaine. On transfère ainsi la validité d'universaux biologiques de l'espèce humaine à la sphère des représentations et comportements individuels. 
De telles méthodes paraissent pertinentes pour les problématiques managériales soustendues par la psychologie des individus, notamment en marketing et en ressources humaines. Mais que penser du décalque de ces méthodes à l'étude des représentations et comportement collectifs des organisations ? Les chercheurs en gestion qui s'y livrent ne commettent-ils pas la même erreur que celle dénoncée par Piketty à propos des théories à agent représentatif? Quelle rigueur scientifique attribuer aux travaux qui entendent traiter les organisations elles-mêmes comme des personnes déchiffrables par les outils de la psychologie quantitative ? Les affirmations épistémologiques de Piketty sont la dénonciation d'un certain obscurantisme académique en sciences humaines, qui a contaminé le champ du management de longue date. En témoignent les construits obscurs que sont, parmi de nombreux exemples, la «capacité d'absorption» ou encore les «capacités dynamiques $»^{4}$ des organisations vues comme des personnes, qui détournent notre champ des problèmes importants pour les managers comme pour les acteurs sociétaux.

Troisièmement, et ceci fera transition avec la question du supposé marxisme de Piketty, l'ouvrage dénonce et démontre la nocivité des indicateurs utilisés par les économistes pour mesurer les inégalités. Par exemple, le découpage des populations en déciles de richesse n'est pas assez fin pour détecter l'actuelle divergence des super-riches et revient à l'effacer des dossiers de référence comme ceux de l'OCDE : "Ces rapports sont censés contribuer à informer le débat public sur la répartition des richesses, et en pratique ils présentent souvent une vision artificiellement apaisée des inégalités. » (p. 420). De même, le célèbre indicateur de Gini, censé mesurer objectivement le degré d'inégalité d'une société, contribuet-il selon Piketty au déni de la divergence en cours, en ce qu'il ne sépare pas les contributions respectives de la rente et du travail, occultant ainsi le retour à une société d'héritier: "Les indicateurs synthétiques tels que le coefficient de Gini donnent une vision abstraite et aseptisée de l'inégalité, qui non seulement ne permet pas aux uns et aux autres de se situer dans la hiérarchie de leur temps [...]» et notamment «pour les économistes qui ont tendance à oublier qu'ils sont dans le centile supérieur » (p. 420).

\section{Un geste commun au marxisme : la volonté de démystifier...}

Dans sa volonté de soulever le «voile pudique » (p. 420) qui recouvre la Grande Divergence $^{5}$ en cours, Piketty reprend quelque chose du geste marxiste, si l'on en croit ce que les thuriféraires historiques de Marx en disent par eux-mêmes ([1965] 2005, p. 80) : «Si le "chemin de Marx" est exemplaire, c'est non par ses origines et son détail, mais par sa volonté farouche de se libérer des mythes qui se donnaient pour la vérité, et par son rôle de l'expérience de l'histoire réelle qui a bousculé et balayé ces mythes. » En particulier, la volonté de rendre visible ce qui a été occulté par la science économique ne serait pas désavouée par les Marxistes historiques.

5. Sur cette dernière notion voir le résumé proposé par Cohendet et al. (2014).

6. Nous assumons la paternité de cette expression. 
On pourrait dire du travail de Piketty ce qu'Althusser disait dans Lire le Capital (Althusser et al., [1965] 1996, p. 14) : «Comprendre cette identité nécessaire et paradoxale du non-voir et du voir dans le voir même, c'est très exactement poser notre problème ». Néanmoins, nous avancerons que c'est cela, et cela seulement qui autorise un rapprochement entre les deux démarches : la volonté de démystifier une économie considérée comme allant de soi, ainsi que le séisme intellectuel que provoque cette démystification.

\section{3. ...mais en aucun cas une théorisation ni une épistémologie marxistes}

Certes Piketty reconnaît l'importance de ce qu'il considère être la contribution majeur de Marx : l'énonciation du «principe d'accumulation infinie » du capital : " c'està-dire la tendance inévitable du capital à s'accumuler et à se concentrer dans des proportions infinies, sans limite naturelle. » (p. 28). Mais sous deux aspects majeurs le travail de Piketty se sépare radicalement de celui de Marx.

Sur le plan théorique, Piketty tient à se démarquer de l'œuvre marxienne. En dehors du principe d'accumulation infinie, il constate en premier lieu que l'apocalypse marxiste ne s'est pas réalisée, en raison des gains de productivité et de la croissance démographique qui ont compensé l'accumulation du stock de capital, infirmant ainsi à long terme la baisse tendancielle des taux de profit. Ce point est cristallisé par l'équation asymptotique $\beta=\mathrm{s} / \mathrm{g}$ exposée plus haut: «Pour résumer: la croissance moderne, qui est fondée sur la croissance de la productivité et la diffusion des connaissances, a permis d'éviter l'apocalypse marxiste et d'équilibrer le processus d'ac- cumulation du capital. Mais elle n'a pas modifié les structures profondes du capital ou tout du moins elle n'a pas véritablement réduit son importance macroéconomique relativement au travail. » (p. 370-371). Plus sévères encore sont les critiques formulées sur un plan méthodologique. Tout d'abord, explique Piketty, la base empirique des travaux de Marx est fragile : « Le problème est qu'en dépit de toutes ces intuitions importantes Marx conserve le plus souvent une approche relativement anecdotique et peu systématique des statistiques disponibles » (p. 363). Ensuite, Marx est jugé obscur dans son propos : Marx n'utilise pas de modèle mathématique, et sa prose n'est pas toujours limpide, si bien qu'il est difficile de savoir avec certitude ce qu'il avait en tête » (p. 360). Ces constats apparaissent rétrospectivement plus cruels encore pour les auteurs estampillés marxistes que pour Marx lui-même, en ce qu'ils sous-entendent que les millions de pages écrites à propos du Capital pourraient n'être que des gloses fantasmagoriques produites sur la base de textes ambigus. Un constat que les marxistes de la fin du $\mathrm{XX}^{\mathrm{e}}$ siècle ont fini par admettre eux-mêmes. Ainsi Wallerstein, expliquait-il lui-même que « Nous devrions prendre au sérieux son affirmation [de Karl Marx] selon laquelle il n'était pas marxiste, et non l'écarter comme un simple bon mot. " ([1983] 2011, p. 10). De même, le grand-prêtre du marxisme structuraliste qu'était Althusser avait-il fini par écrire (1994, cité par Garo in Bourdin, 2008, p. 33) : «Je peux dire qu'en un certain sens, Aron avait un peu raison. Nous avons fabriqué une philosophie "imaginaire" pour Marx, une philosophie qui n'existait pas dans son œuvre, si l'on s'en tient strictement à la lettre de ses textes. » 
Ce constat nous conduit à examiner dans quelle mesure Piketty se distingue philosophiquement et épistémologiquement de Marx. On peut relever entre les deux auteurs au moins deux points de disjonction majeurs. Le premier et le plus évident est le rôle globalement mineur accordé par Piketty aux relations de production (ce que ne manquent d'ailleurs pas de lui reprocher certains de ses collègues économistes, $\mathrm{cf}$. notre introduction). Un marxiste contemporain étudiera les nouvelles conditions de production et, sur cette base esquissera les contours du nouveau monde à venir. C'est ce qu'entreprend par exemple Yann Moulier-Boutang dans son ouvrage sur le « capitalisme cognitif » (2007). À l'opposé, Piketty considère la richesse quelle que soit la nature de son support. La rente foncière et son nouvel avatar, la rente immobilière, sont pour lui aussi important que le capital productif dans la dynamique de la Grande Divergence en cours. Un deuxième critère de démarcation par rapport au marxisme réside dans le rôle accordé à l'idéologie. En soulevant le voile d'ignorance qui recouvre la divergence entre les centiles les plus riches et le reste de la population, Piketty entend ouvrir un débat public, et semble vouloir éclairer la discussion politique en vue d'infléchir le futur. À l'opposé, les marxistes historiques ont toujours considérés que ce genre de débats, confinés à la « superstructure » de la société, ne permettaient pas de transformer le monde, si bien que seule un bouleversement révolutionnaire de l' « infrastructure » économique pourrait faire la différence. Plus profondément, la pensée du soupçon qui sous-tend le marxisme est si radicale que, bien souvent, toute pensée y est vue comme relevant de l'idéologie. Pour Althusser par exemple (Althusser, (1965) 2005, p. 238), le concept d'idéologie est très général : « l'idéologie est un système [...] de représentations (images, mythes, ou concepts selon les cas) doué d'une existence d'un rôle historiques au sein d'une société donnée. » Cette extrême extensivité du concept d'idéologie la fait considérer comme une dimension inévitable de toute expérience sociale : " L'idéologie n'est donc pas une aberration ou une excroissance contingente de l'Histoire : elle est une structure essentielle à la vie des sociétés » (ibid., p. 239), au point que même les scientifiques dans un monde parvenu un jour au stade du communisme ne sauraient y échapper : « le matérialisme historique ne peut concevoir qu'une société communiste elle-même puisse jamais se passer d'idéologie ». Piketty est à des années-lumière de ce genre de position quant aux rôles des savants, de la pensée et du débat public dans la société. À l'opposé des penseurs du soupçon, il croit en la Raison, ce qui le rapproche infiniment plus de la pensée des Lumières et de la foi kantienne en la raison (certes limitée) que de toute variété de marxisme ou philosophie marxiste. Sa manière de poser les problèmes est tout autre, et son travail transdisciplinaire s'inscrirait dans l'appel de Rosanvallon (2011, p. 354): «L'enjeu est de constituer une économie politique élargie du lien social, qui permette de fonder une véritable théorie générale de l'égalité intégrant ses différentes dimensions afin de donner des bases solides et universalisables aux actions réformatrices. " Pour résumer, ce travail fait de Piketty un penseur des Lumières à l'opposé des obscurités marxistes et a fortiori des ténèbres bolcheviques. 


\section{CONCLUSION : VERS DE NOUVELLES LUMIÈRES... OU DE NOUVELLES TÉNĖBRES?}

Peut-être la magnitude du séisme pikettien sera-t-elle appréciée à une « juste valeur » transdisciplinaire si on la met en perspective avec l'ouvrage de même focale produit par un historien disciple de Braudel trente ans auparavant. Dans Le capitalisme historique, Immanuel Wallerstein (1983) proposait une définition elle aussi centrée sur le mécanisme d'accumulation: "Le capitalisme historique désigne donc le système social où se sont trouvés à la fois élargi sans interruption le champ d'exercice des lois de l'accumulation (ce qu'on appelle parfois le «loi de la valeur»), renforcée la sévérité avec laquelle s'exerçaient ces lois de l'accumulation, accru sans cesse le degré de pénétration de ces dernières dans la "machine sociale", alors même que dans la société l'opposition à ces règles haussait le ton et s'organisait. » (p. 17). À l'instar de Marx, Wallerstein avait formulé sa propre prophétie : «Il ne faut pas oublier que les systèmes historiques sont précisément historiques. Ils viennent au jour et finissent par disparaître à la suite de processus internes au cours desquels l'exacerbation de leurs contradictions internes conduit à une crise structurelle. Les crises structurelles se distinguent par leur caractère massif et leur durée. Leur développement est lent. Le capitalisme historique est historiquement entré dans une crise structurelle de ce genre dans les premières années du $\mathrm{XX}^{\mathrm{e}}$ siècle et il connaîtra sans doute sa fin comme système historique au cours du suivant. » (1983, p. 92-93). À l'aube de la révolution néolibérale, Wallerstein localisait la dynamique de divergence essentielle entre pays développés et pays en développement (p. 105) : « un accroissement de l'écart entre le surplus consommé par les 10 à $15 \%$ les plus favorisés de la population mondiale et celui consommé par le reste de l'économiemonde », et il affichait une position résolument pessimiste (p. 128) : "Le système actuel ne peut pas continuer à exister ; il est en train d'épuiser ses mécanismes d'ajustement. Mais ce qui va en sortir, le nouveau système-monde (ou les nouveaux systèmesmondes), ne peut pas être prédit. »

Le capital au XXI e siècle se situe pour une bonne part en orthogonalité avec les prophéties de Wallerstein. Tout d'abord Piketty prévoit une homogénéisation relative à l'échelle mondiale là où Wallerstein voyait s'accroître les écarts. Mais surtout, là où ce dernier voyait dans les crises du $\mathrm{XX}^{\mathrm{e}}$ siècle les signes d'une crise terminale du capitalisme, le premier y diagnostique au contraire un rajeunissement qui a permis de repousser, en ruinant les rentiers, la venue de la Grande Divergence. Le point de rencontre des deux pensées se situe dans la prévision de bifurcations majeures au cours du XXI ${ }^{\mathrm{e}}$ siècle. À peine se sont-elles croisées, toutefois, que les deux pensées s'écartent largement l'une de l'autre. Pour Piketty, ce sont la démocratie et la citoyenneté qui sont en danger, et non le capital, dont la machine infernale fait tourner plus fort que jamais l'accumulation et la concentration de la richesse.

Là encore il est intéressant de constater à quel point les études de longue durée, lorsqu'arrive le moment de la synthèse, sont sensibles aux effets d'optique provoqués par l'échantillonnage temporel, un peu comme ces images que les lentilles 
inversent lorsque leur focale se déplace. En inversant la courbe de Kuznets par une simple dilatation des durées observées, Le Capital au XXIe siècle est dans son cœur même plongé dans des jeux d'inversions optiques de ce genre.

À notre tour d'en proposer notre propre inversion de focale, en espérant nous tromper : il apparaît en effet une curieuse analogie entre la démarche de Piketty et le geste intellectuel marxiste, par-delà leurs différences de contenu. Feuerbach ([1841] 1992) voulait en effet opérer un renversement de la dialectique hégélienne et Marx opéra un renversement de ce renversement dans ses Thèses sur Feuerbach (Macherey, 2008). Il semble de même que Piketty inverse l'histoire du capitalisme narrée par Braudel et son disciple Wallerstein: le $\mathrm{XXI}^{\mathrm{e}}$ siècle ne sera peut-être pas celui de l'extinction du capitalisme, mais celui d'un système-monde encore plus féroce.
Mais le champ de l'avenir ouvre également à spéculer sur un renversement de ce renversement par déplacement de la focale temporelle à plus grande échelle encore : l'extinction du capitalisme prédite par Wallerstein peut se réinscrire, en mettant l'œuvre de Piketty en perspective, dans un cycle de cycles encore plus vaste où les 500 dernières années n'apparaîtraient que comme la répétition préliminaire d'un bien plus grand jeu de concentration et d'oppression, dans un monde désormais fini que ne viendrait faire respirer aucune marge extérieure ni civilisation alternative. Un nouvel âge de fer et d'oppression comme le fut le $\mathrm{XVII}^{\mathrm{e}}$ siècle du premier capitalisme naissant, sous contraintes de ressources finies et de changement climatique. L'amplitude potentielle de ces jeux d'optique ne peut qu'en retour encourager la communauté des chercheurs en gestion à rechercher toujours plus de réflexivité sur leurs propres travaux.

\section{BIBLIOGRAPHIE}

Althusser L. ([1965] 2005). Pour Marx, Éd. La Découverte, Paris, France.

Althusser L., Balibar E., Establet R., Macherey P., Rancière J. ([1965] 1996). Lire Le Capital, Ed. Presses Universitaires de France, coll. « Quadrige », Paris, France.

Balibar E. (1993), La philosophie de Marx, Éd. La Découverte, Paris, France.

Baverez N. (2013). «Piketty, un marxisme de sous-préfecture », Le point, 26/09/2013, http://www.lepoint.fr/editos-du-point/nicolas-baverez/piketty-un-marxisme-de-sous-prefe cture-26-09-2013-1735260_73.php

Bourdin J.-C. (coord.) (2008). Althusser : une lecture de Marx, Éd. Presses Universitaires de France, coll. « Débats ».

Boyer R. (2013). « Le capital au XxI ${ }^{\mathrm{e}}$ siècle », Revue de la régulation [Online], $14 \mid 2^{\mathrm{e}}$ semestre / Autumn 2013, Online since 12 December 2013. URL : http://regulation.revues.org/10352

Chavagneux C., Jeanneau L. (2014). «Inégalités : l'onde de choc Piketty », Alternatives économique, $\mathrm{n}^{\circ} 336$, juin, p. 59-68. 
Cohendet P., Mehouachi C. et Simon L. (2014). « Connaissance et Innovation », Encyclopédie de la stratégie, Tannery F., Denis J.-P., Hafsi T. et Martinet A.C. (dir.), Vuibert.

Garo I. (2008). «La coupure impossible. L'idéologie en mouvement, entre philosophie et politique dans la pensée de Louis Althusser», Althusser: une lecture de Marx, Bourdin J.-C. (coord.), Éd. Presses Universitaires de France, p. 31-55.

Husson M. (2014). «Le capital au XXIe siècle, Richesses des données, pauvreté de la théorie », Contretemps, 10 février 2014.

Feuerbach L. ([1841] 1992). L'essence du Christianisme (das Wesen des Christenthums), Gallimard, Paris, préface et traduction by J-P. Osier.

Foucault M. ([1984] 1994). «Qu’est-ce que les Lumières », Dits et Écrits (1994), Gallimard, Paris, p. 679-688.

Fukuyama F. (1992). The End of History and the Last Man, Free Press, ISBN 0-02-910975-2.

Fukuyama F. (1989). “The End of History?”, The National Interest (Summer 1989).

Landais C. Piketty T., Saez E. (2011). Pour une révolution fiscale. Un impôt sur le revenu pour le XXI siècle, Éditions du Seuil, coll. « La République des Idées ».

Macherey P. (2008). Les "thèses" sur Feuerbach: traduction et commentaire, Éd. Amsterdam, paris, France.

Moulier Boutang Y. (2007). Le capitalisme cognitif - La Nouvelle Grande Transformation, Éd. Amsterdam, coll. « Multitudes/Idées », Paris, France.

Piketty T. (2013). Le Capital au XXIe siècle, Éditions du Seuil, Paris; Annexes en lignes http///piketty.pse.ens.fr/capital21c; Traduction anglaise (2014) Capital in The Twenty-First Century, Ed. The Belknap Press of Harvard University Press, Cambridge, Massachussets.

Rosanvallon P. (2011). La société des égaux, Éditions du Seuil, Paris.

Wallerstein I. ([1983] 2011). Le capitalisme historique, Éd. La Découverte, Paris, France.

Zimnovitch H. (2014). « Le capital au XXIe siècle, Thomas Piketty, », Comptabilité Contrôle Audit, avril, vol. $20 \mathrm{n}^{\circ} 1$, p. 122. 
